\title{
Response of a Sodium-Iodide Scintillation Spectrometer to 10- to 20-Million-Electron-Volt Electrons and X-Rays ${ }^{1}$
}

\author{
H. W. Koch and J. M. Wyckoff
}

\begin{abstract}
The response of a large-crystal sodium-iodide spectrometer was studied for individual monoenergetic electrons extracted from a 50-million-electron-volt betatron operated between 1 and $20 \mathrm{Mev}$. The energy resolution with crystals 5 inches in diameter by 4 inches in length and 5 inches in diameter by 9 inches in length reaches its optimum value for electrons of $6 \mathrm{Mev}$, where the total width of the pulse-height distribution at half maximum is 4 percent. Syntheses of pulse-height distributions for 11- and 19-Mev monoenergetic X-ray photons are made by using the experimental electron pulse-height distributions. The computed results compare favorably with the measured pulse-height distributions for 11.6- and 17.6-Mev gamma rays. Syntheses provide one of the few procedures for predicting spectrometer response functions in this $\mathrm{X}$-ray energy range.
\end{abstract}

\section{Introduction}

The pulse-height distributions produced in totalabsorption spectrometers by monoenergetic X-rays show a narrow peak with small tail at low pulse heights [1]. ${ }^{2}$ These spectrometers are therefore suitable for the accurate study of continuous distributions of X-rays and for analyses of the shape of the continuum. However, an analysis of the spectrometer output in terms of the number of X-ray photons at each photon energy requires detailed information about the response function of the spectrometer to monoenergetic $\mathrm{X}$-rays or gamma rays in the energy range of interest.

For energies below $3 \mathrm{Mev}$, gamma rays from long-lived radioactive sources are available at sufficiently small energy intervals to permit the accurate evaluation of the spectrometer response.

At energies above $3 \mathrm{Mev}$, the available nuclear gamma rays are few, weak in intensity, and generally unsuitable for the determination of response functions. For example, the relatively monoenergetic $\mathrm{Li}^{7}(p, \gamma) \mathrm{Be}^{8}$ gamma rays are a $17.6-\mathrm{Mev}$ gamma ray accompanied by a broad line at $14.8 \mathrm{Mev}$. The lower energy line is sufficiently broad as to obscure somewhat the interpretation of the shape of the 17.6- $\mathrm{Mev}$ pulse-height distribution. Similarly, the $\mathrm{B}^{11}(p, \gamma) \mathrm{C}^{12}$ and the $\mathrm{Be}^{9}(\alpha, n \gamma) \mathrm{C}^{12}$ reactions provide 11.6- and 4.43-Mev gamma rays, respectively, that are weak in intensity, and experiments with them are consequently troubled by large relative backgrounds.

The response function can also be obtained by a Monte Carlo calculation [2]. However, these calculations are tedious and very time-consuming even when performed with the aid of automatic computers. The difficulty arises from the multiple interactions that must be followed in some detail at energies above $3 \mathrm{Mev}$.

In order to provide some detailed data on the response of a large-crystal spectrometer for 10- to 20Mev monoenergetic X-rays, the response to monoenergetic electrons was examined [3] as described in this report, and then the pulse-height distributions for

1 This research was supported by the United States Air Force, through the Office of Scientific Research of the Air Research and Development Command.

${ }_{2}^{2}$ Figures in brackets indicate the literature references at the end of this paper. two X-ray energies were synthesized from these data. Specific X-ray energy values of 11 and $19 \mathrm{Mev}$ were chosen for the synthesis on the basis of a planned matrix covering the 10 - to $20-\mathrm{Mev}$ region. These values are also reasonably near experimentally measured monoenergetic gamma-ray peaks of 11.6 and $17.6 \mathrm{Mev}$, and so the calculations lend themselves to a comparison with the experimental results.

The sodium-iodide crystals in the scintillation spectrometer used in the electron part of the experiment were 5 -in. diameter by 4 in. long, 5 in. diameter by 5 in. long, and 5 in. diameter by 9 in. long. Pulse-height distributions were obtained for electrons with energies between 1 and $20 \mathrm{Mev}$. The results gave the shapes of the distributions, the variation of the relative resolution (full-width at half maximum divided by the pulse height at the maximum) with electron energy; and the linearity of the pulse height of the peak of the pulse-height distribution versus electron energy.

The pulse-height distributions for monoenergetic X-rays were synthesized for a 5-in.-diameter by 9-in.long-crystal spectrometer from the electron results. This work, which is also described in this report, was motivated by the fact that good predictions of pulseheight distributions produced by X-rays are difficult to obtain. By using the experimentally determined pulse-height distributions for electrons with energies above $6 \mathrm{Mev}$, one removes the necessity for following the electron through its detailed interactions, as would ordinarily have to be done in a complete calculation.

\section{Response of the Spectrometer to Electrons}

The electron beam was removed from a $50-\mathrm{Mev}$ betatron by a pulsed electromagnetic extractor [4]. Individual electrons with fixed energies in the range 1 to $20 \mathrm{Mev}$ were directed against the center of the flat surface of the sodium-iodide crystal, as shown in figure 1 .

Electrons were available from the extractor during a time interval of approximately $0.2 \mu$ sec every $5,500 \mu \mathrm{sec}$. The electrons from the betatron should be monoenergetic to at least 0.1 percent over periods 


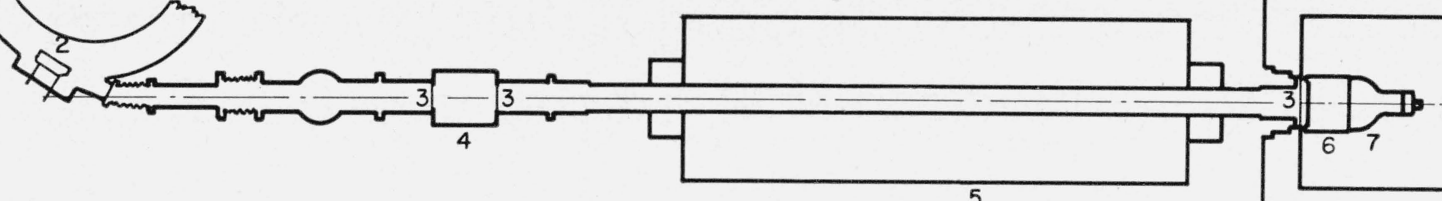

Figure 1. Experimental arrangement.

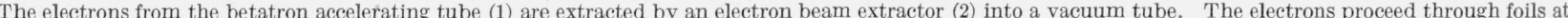

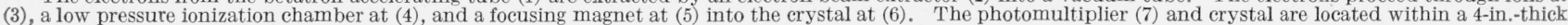
lead shield (8).

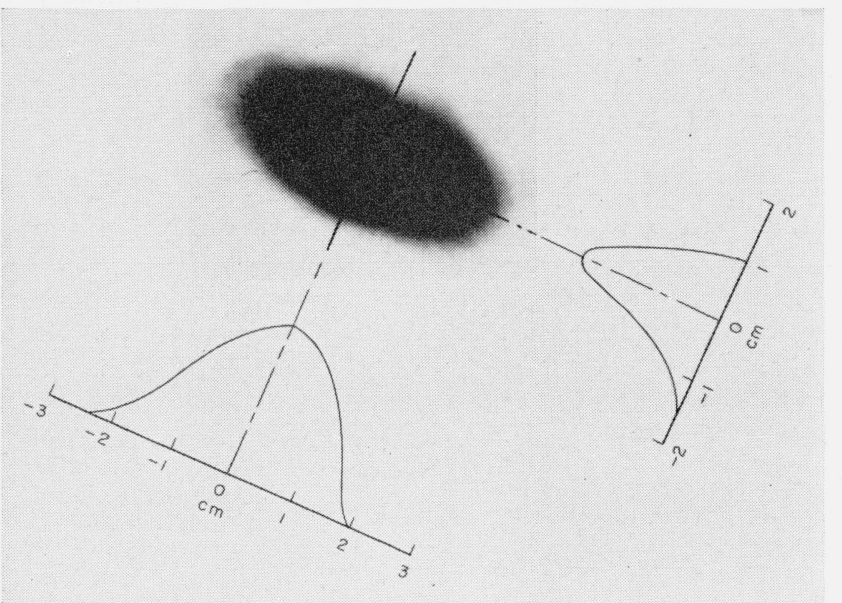

Figure 2. The electron beam at the exit of the vacuum tube.

The intensity curves shown were taken from a film with a densitometer. The electron beam current for this picture was of the order of $10^{-10}$ amperes.

Figure 3. Pulse-height distributions produced by electrons from a betatron in a 5-in.-diameter by 5-in.-long sodiumiodide spectrometer.

The electron energies given on each curve were determined from an energy comparison of the peak pulse heights with those obtained with 1.12- or 4.43 Mev gamma rays.
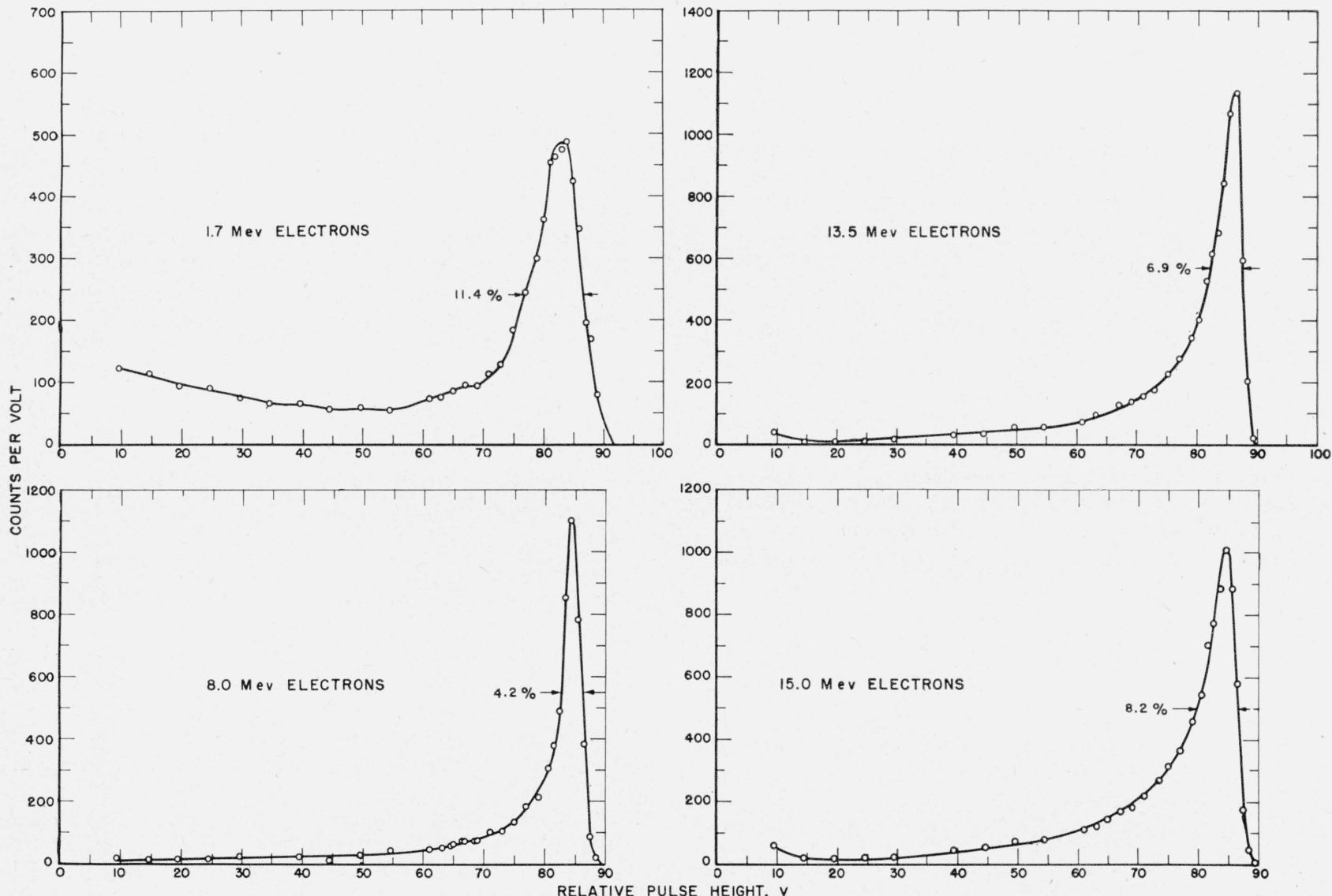
of time of the order of minutes [5]. Energy straggling of the electrons produces the largest uncertainty in the energy in the present experiment because of the three 0.0014 -in. aluminum vacuum windows and the aluminum-magnesium-oxide entrance window of the crystal container. The most probable energy loss is $300 \mathrm{kev}[5]$, and the energy uncertainty is less than $100 \mathrm{kev}$.

Adjustments on a beam of $10^{9}$ electrons/sec were made to direct the electrons down the collimator hole and to set the focusing magnet current without the spectrometer. The electron-beam intensity was then adjusted so that the counting rate with the spectrometer was limited to five electrons detected per second. Since 180 bursts of electrons come from the betatron in $1 \mathrm{sec}$, the coincidence detection of electrons during any one betatron burst was limited to about 3 percent of all electrons detected. A picture of the electron-beam size at the crystal position is shown in figure 2.

The individual light pulses in the sodium-iodide crystals were detected by the photomultiplier-amplifier arrangements previously described [1]. The output pulses from the linear amplifier were sent into a gain-of-two amplifier and into a 30-channel differential analyzer. Two separate runs at each electron energy were made in order to check, first, the over-all shape of the pulse-height distribution, and, second, the detailed shape of the peak. The two runs were separated and followed by calibration runs with a mercury-relay precision sliding pulser and a radioactive gamma-ray source. The $4.43-\mathrm{Mev}$ gamma rays from a $\operatorname{RaD}(\alpha, \mathrm{Be})$ source or the 1.12 Mev gamma rays from $\mathrm{Zn}^{65}$ were used as calibration sources.

The betatron energy control developed by Saunders [6] in conjunction with the beam extractor arrangement was used to produce electrons with energies of $1.22,1.96,4.04,5.12,6.18,8.30,12.2,13.8,15.3$, 17.15 , and $17.9 \mathrm{Mev}$, respectively. The energy of the electrons entering the crystal was assumed to be $300 \mathrm{kev}$ less than these values.

Typical pulse-height distributions for 1.7-, 8.0-, 13.5-, and 15-Mev electrons entering the 5-in.-diameter by 5-in.-long sodium-iodide crystal are shown in figure 3.

Results of the percentage resolution as a function of the incident electron energy are given in figure 4.

The resolution for electrons of energy less than 6 $\mathrm{Mer}$ deviates markedly from that expected on the basis of the statistical fluctuations in the number of photons detected by the photomultipliers [1]. This was due to the scattering and straggling in the crystal entry window, which are important at low energies. At about 6 or $7 \mathrm{Mev}$ the resolution values are those one would expect from the statistical fluctuations. Because the synthesis of pulse-height distributions for X-rays that follows depends on pulse height distributions obtained for electrons liberated on the crystal axis and within the crystal, the energy resolution values used for electron energies less than 6 Mev were those assumed to be determined by statistical fluctuations with a Gaussian half-width inferred from previous work with a similar spectrom-

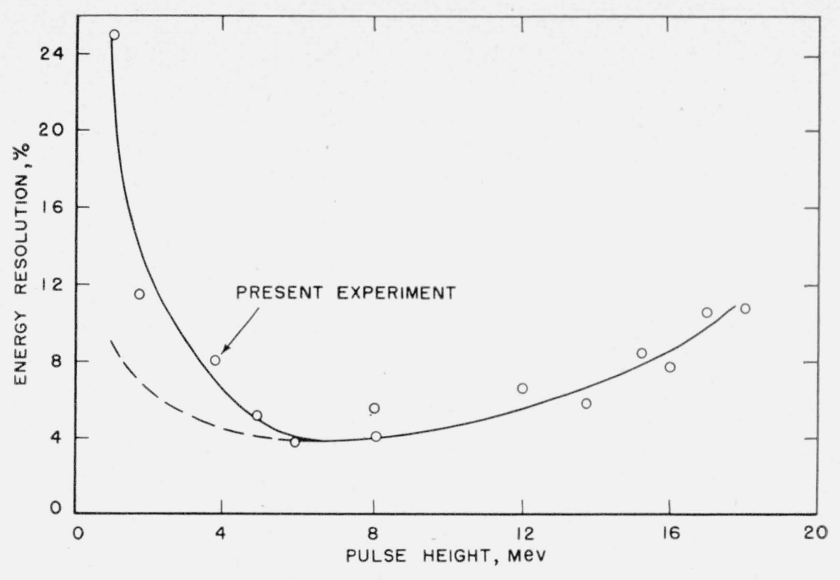

Figure 4. Percent energy resolution inferred from curves of the type shown in figure 3.

The lower curve from 1 to $6 \mathrm{Mev}$ was derived from earlier data [1] with gamma rays.

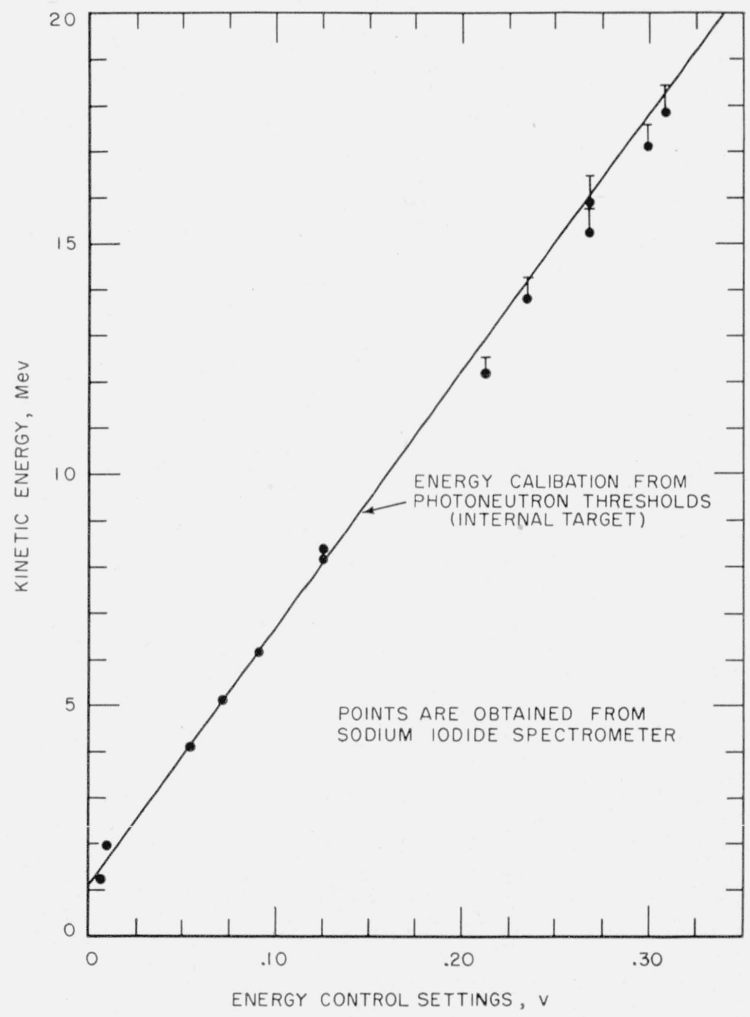

FIGURE 5. Energy calibration of the betatron with the spectrometer data.

The points are located at energies determined by gamma-ray energy calibrations plus a most probable energy loss correction due to foils between the betatron and the spectrometer crystal. The vertical lines above the experimental points indicate the possible error due to an assignment of energy by a comparison
of the peak pulse-height positron in the electron distributions and the low-energy of the peak pulse-height po
gamma-ray distributions.

eter [1]. For electron energies above $6 \mathrm{Mev}$, the experimental distributions similar to those in figure 3 were used.

Figure 5 provides a crude test of the linearity of the spectrometer response to electron energy. The ordinate is the kinetic energy of the electrons. The 
abscissa scale is the potentiometer setting in volts on the betatron energy control [6] to which the electron energy has been shown to be related by a linear function. The experimental points are the energy values assigned to the pulse heights of the peaks in figure 4 plus $300 \mathrm{kev}$. This assignment was made by a comparison with a known gamma-ray energy of 4.43 or $1.12 \mathrm{Mev}$.

Tests were also made with a 5-in.-diameter by 4-in.-long crystal and a 5-in.-diameter by 9-in.-long crystal. No significant departures in distribution shapes or resolution values were found for the different sized crystals. It was found that only 10 percent of the $15.6-\mathrm{Mev}$ electrons produced a contribution in the last 4 in. of the 9-in.-long crystal arrangement. This contribution consisted of very small pulse heights. It was inferred from these tests that the energy loss from the three sizes of crystal used in this experiment is predominantly due to energy escaping from the crystal sides.
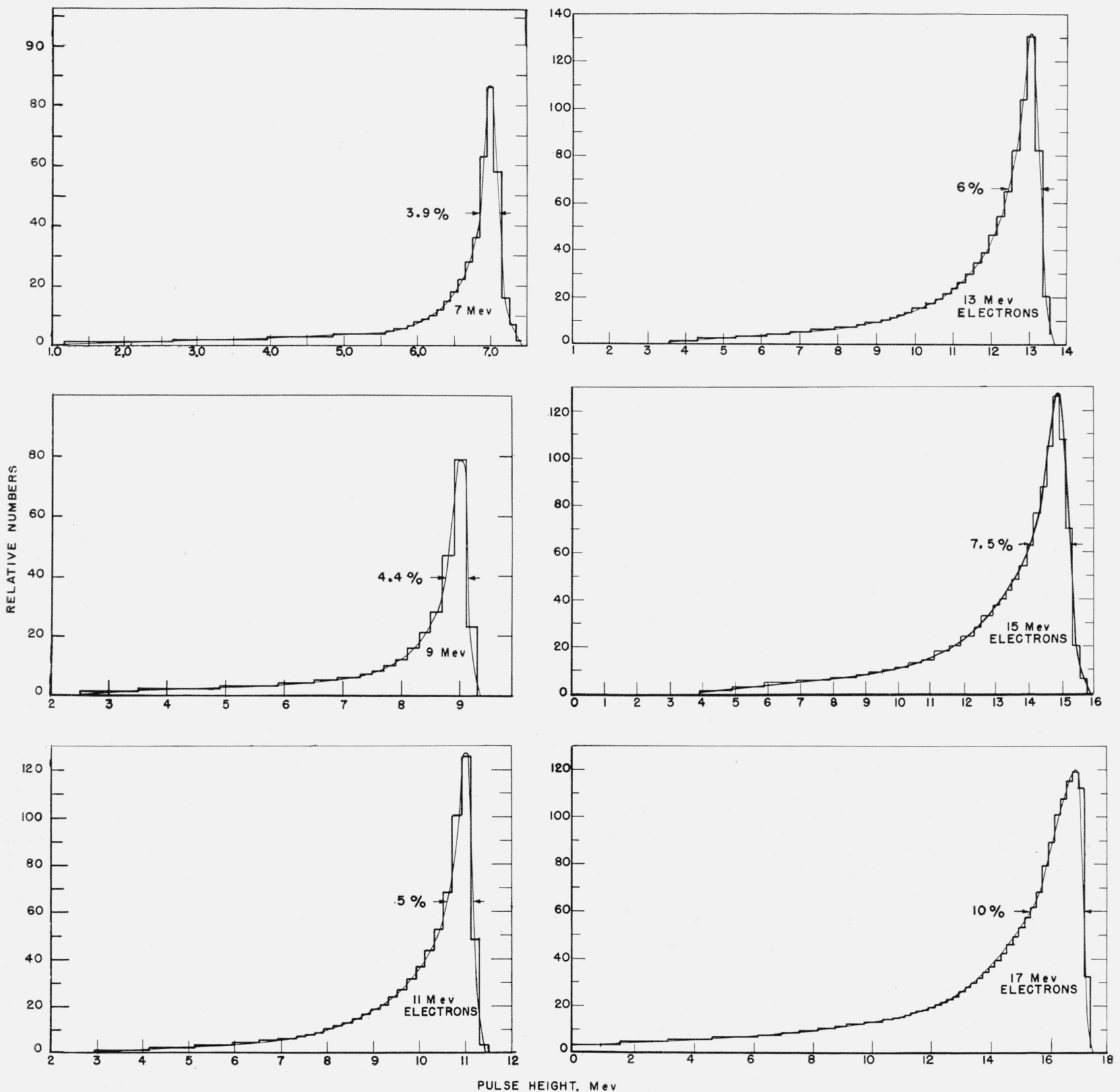

Figure 6. Histograms representing the pulse-height distributions for 7-, 9-, 11-, 13-, and 17-Mev electrons.

The 5-Mev histogram is not shown. It was a Gaussian with a small tail at small pulse heights. The 1- and 3-Mev distributions were assumed to be Gaussian with a percent half-width inferred from the lower curve of figure 4 . 


\section{Synthesis of X-Ray Pulse-Height Distribu- tions}

\subsection{Pair Electron Contributions}

The most important contribution to the pulseheight distribution of X-rays above $10 \mathrm{Mev}$ results from the energy loss in the crystal produced by the pair electrons. Specifically, at $11 \mathrm{Mev}, 68$ percent and at $19 \mathrm{Mev}, 84$ percent of the initial interactions will be by the pair production process.

The origin of the electron pairs produced in the 5 -in. by 9 -in. crystal will be distributed along the axis of the crystal if the X-rays are assumed apertured into a narrow beam that is directed along the axis. If it is further assumed that the electrons and positrons behave sufficiently identically in their scattering and energy-loss characteristics, except for the production of annihilation radiation by the positrons, the resulting pulse-height distributions can be predicted by folding together the measured distributions described in the last section for those electron energies that could combine to form a pair. The combined electron energy would be the incoming X-ray energy minus twice the electron rest-mass energy.

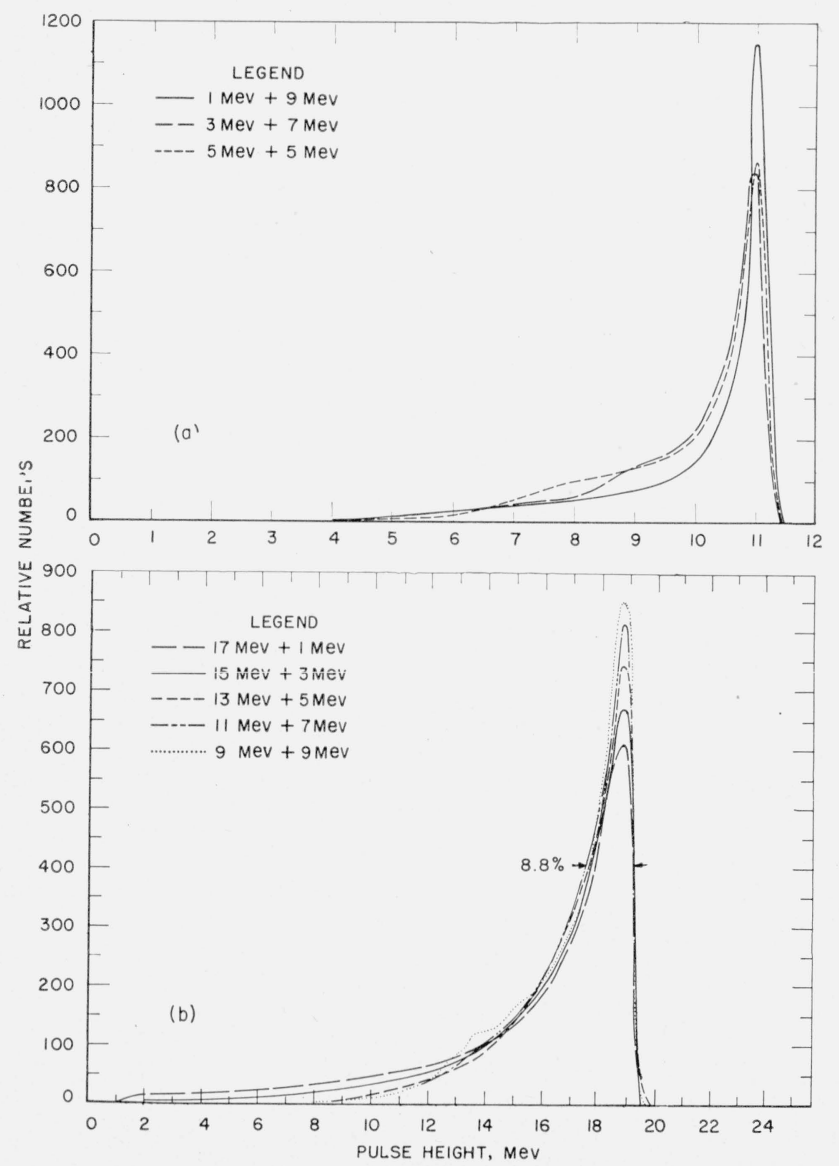

Figure 7. Folded pulse-height distributions for various combinations of pair electron energies.

The structure at small pulse heights is not significant and resulted from the detailed, assumed shapes of the histograms of figure 6 .

(a) Total energy of the two electrons was assumed to be $11 \mathrm{Mev}$.
There are obvious differences between the test electrons from the betatron and the pair electrons produced by an absorption of an X-ray photon. One difference is the angular orientation of the electrons with respect to the crystal axis. If either of the two electrons of a pair has an energy less than $6 \mathrm{Mev}$, the probability that it will lose energy by bremsstrahlung is small. Therefore, the knowledge of the angles into which the electrons are emitted is unimportant, because the electrons will lose energy by collision loss and produce a Gaussian-type pulseheight distribution (see fig. 4 and its discussion above). On the other hand, if the electron energies are greater than $6 \mathrm{Mev}$, the electrons will be projected into angles less than $10^{\circ}[7]$. For the present purposes, these angles are small enough to allow one to assume production of all pair electrons parallel to the crystal axis.

Another difference between test and pair electrons is the crystal depth to the point of initial interaction. The test electrons interact as soon as they enter the crystal, whereas the pair electrons interact after the depth of X-ray absorption. However, for the 5-in.diameter by 9-in.-long crystal, 85 percent of the photons interact in the first 5 in. of the crystal. Also very little effect of length from 4 to 9 in. was detectable in the electron experiment described above. Therefore, there should be little difference in the actual available interaction distance for test and pair electrons.

With the above assumptions, the pulse-height distributions produced by pair electrons were obtained by folding together the pulse-height distributions for electrons whose combined total energy equaled the X-ray energy of interest minus $1 \mathrm{Mev}$. The individual distributions were inferred from the experimental data such as shown in figure 4 , and are drawn as histograms in figure 6 . The results of folding together distributions of electrons with kinetic energies of 1 and $9 \mathrm{Mev}, 3$ and $7 \mathrm{Mev}$, and 5 and $5 \mathrm{Mev}$ are given in figure 7 , a. Similarly, distributions of electrons of 1 and 17,3 and 15,5 and 13,7 and 11 , and 9 and $9 \mathrm{Mev}$ are given in figure 7, b. As all pair electron distributions are equally probable [8], these curves are simply added together, with double weight being given to the 5 plus $5 \mathrm{Mev}$ and the 9 plus $9 \mathrm{Mev}$ curves, respectively. The annihilation radiation contributions are not shown in these curves but are treated separately in part 3.4. These contributions to the final syntheses of the X-ray pulse-height distributions will be shown in figures 9 and 10.

\subsection{Compton Electron Distributions}

The incoming X-rays with energies between 10 and $20 \mathrm{Mev}$ can enter the crystal and undergo Compton scattering as well as produce pairs. In order to include these contributions in the X-ray pulse-height distribution, it has been assumed that the distribution of electron energies for the initial Compton interaction of high-energy photons represents the distribution of the sum of the electron energies after multiple Compton interactions of the same initial photons. 


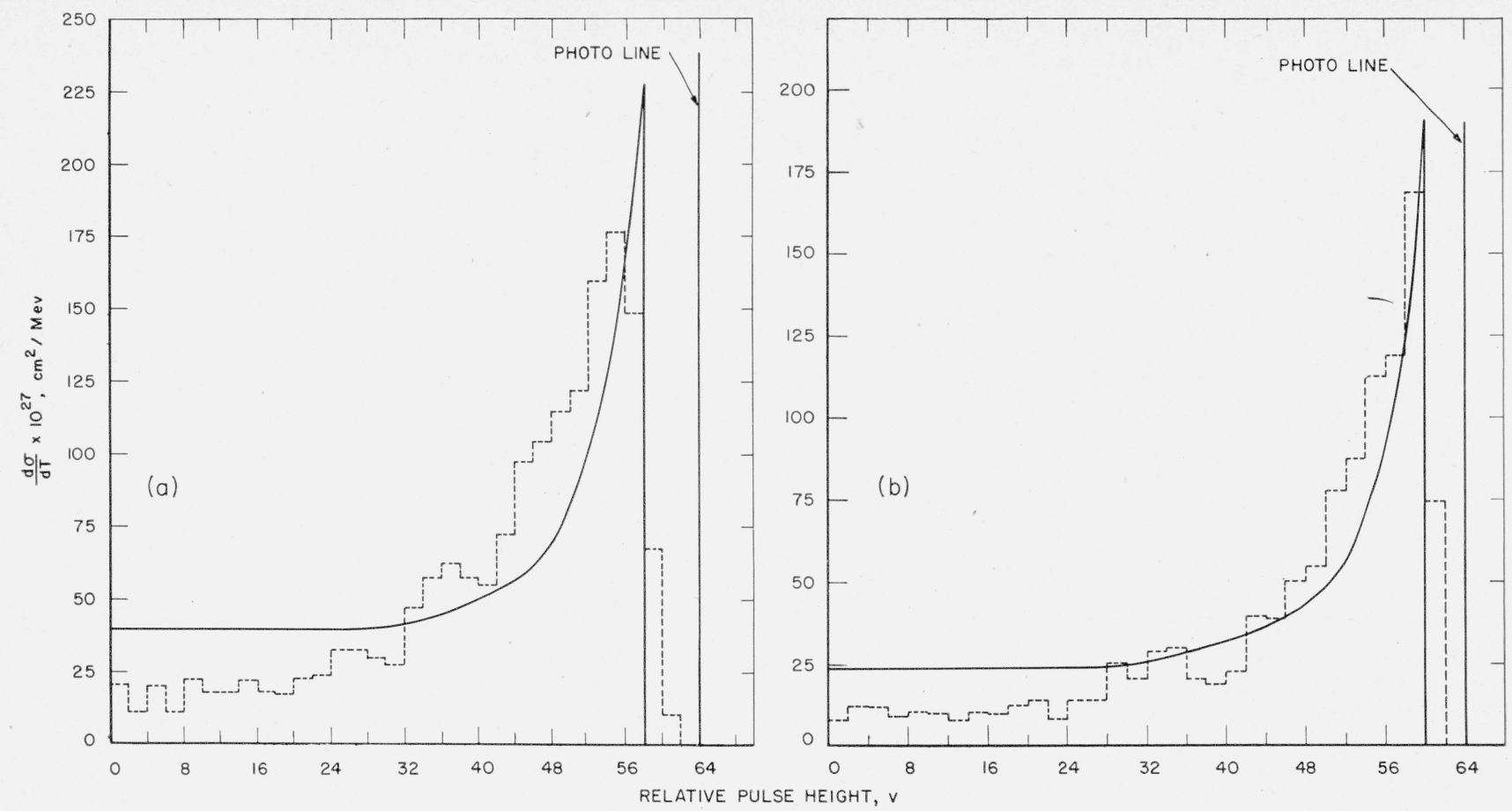

Figure 8. Comparison between Monte Carlo predictions (histograms) and a single-interaction calculation (solid curve) for the Compton electron distributions.

The predictions apply to a 5-in.-diameter by 9-in.-long crystal.

(a) Incident photon energy was $2.62 \mathrm{Mev}$.

(b) Incident photon energy was $4.45 \mathrm{Mev}$.

The justification for this assumption comes from a consideration of the Monte Carlo predictions of Berger and Doggett [2]. One of their results [9] was the predicted distribution of electron energies resulting from multiple interactions of an X-ray photon. The histograms in figures 8 , a and $8, \mathrm{~b}$ are their data, before a Gaussian distribution has been added, for an X-ray energy of 2.6 and $4.4 \mathrm{Mev}$, respectively. Also drawn on these same figures are the Compton electron distributions for a single interaction [10] for the same X-ray energies. The similarity between the single- and multiple-interaction distributions is striking. As the X-ray energy increases, these distributions will become more peaked and more similar.

Because the Monte Carlo calculation and the single-interaction distribution are similar, the theoretical distribution [10] was used to represent the electron energy distribution resulting from the Compton scattering of X-rays in the range from 10 to 20 Mev. This should be a valid assumption especially because the Compton process is a correction and does not predominate in the determination of the shape of the pulse-height distribution for X-rays.

\subsection{Compton Scattering Electron Contribution to the Photoline Energy}

Those Compton scattering events for which the scattered X-ray interacts several times within the crystal, can lead to total absorption of the primary $\mathrm{X}$-ray energy. These events have been included as a
Gaussian-shaped contribution at the photoline energy. (The photopeak itself, arising from photons that have lost all their energy by the photoelectric process, is negligibly small at these energies.) In the 10 - to $20-\mathrm{Mev}$ energy range, the area of this Gaussian was related to the Compton electron distribution by the following extrapolation from lower energies.

The photofraction, $p^{*}$, has been defined [2] as the ratio of the area under the photopeak of the pulseheight distribution, which is represented by a Gaussian, to the area under the entire pulse-height distribution. Two constants characteristic of a particular geometry and energy enter the expression for this ratio. The constant $a$ is that fraction of the total pair production events for which both annihilation quanta are absorbed in the crystal. The constant $b$ is that fraction of the Compton scattered photons that are multiply scattered and totally absorbed in the crystal. In terms of the photoelectric $(\tau)$, pair $(\pi)$, Compton $(\sigma)$, and total $(\mu)$ absorption cross sections, $p^{*}$ is defined as equal to $(\tau+a \pi+b \sigma) / \mu$.

In order to extrapolate values of $b$ to an energy of $19 \mathrm{Mev}, b$ was calculated up to $5 \mathrm{Mev}$ from the data for $a$ and $p^{*}$ from reference 2 . These calculations are shown in table 1 for a 5 -in. diameter by 9-in.-long sodium-iodide crystal. As will be observed from the sixth column, $b$ varies slowly and should continue to vary slowly up to $19 \mathrm{Mev}$ for a 5 -in. by 9 -in. crystal. As the Compton cross section $(\sigma)$ is small and thus the product $b \sigma$ is small, errors due 
TABLE 1. Calculation ${ }^{a}$ of photofraction, $p *$, where $p^{*}=$ $(\tau+a \pi+b \sigma) / \mu ; a=0.633$ [2]; and the sodium-iodide crystal is 5 in. in diameter by 9 in. long

\begin{tabular}{|c|c|c|c|c|c|c|}
\hline$E$ & $\mu$ & $\tau$ & $\pi$ & $\sigma$ & $b$ & $p^{*}$ \\
\hline $\begin{array}{c}\text { Mev } \\
2 \ldots \\
3 . \ldots \\
4 \\
5\end{array}$ & $\begin{array}{c}c m^{2} / g \\
0.0411 \\
.0361 \\
.0347 \\
.0345\end{array}$ & $\begin{array}{l}c m^{2} / g \\
0.0011 \\
.0006 \\
.0004 \\
.0003\end{array}$ & $\begin{array}{r}c m^{2} / g \\
0.0022 \\
.0059 \\
.0096 \\
.0129\end{array}$ & $\begin{array}{l}c m^{2} / g \\
0.0378 \\
.0296 \\
.0247 \\
.0213\end{array}$ & $\begin{array}{r}0.681 \\
.632 \\
.615 \\
.600\end{array}$ & $\begin{array}{r}0.685 \\
.640 \\
.625 \\
.617\end{array}$ \\
\hline
\end{tabular}

a The values of the absorption coefficients $\mu, \tau, \pi$, and $\sigma$ for sodium iodide were taken from $\mathrm{G}$. White of the Bureau (private communication).

TABLE 2. Calculation a of modified photofractions, $P^{*}$, where $P^{*}=(\tau+A \pi+b \sigma) / \mu ; A=0.633$ [2], and the sodium-iodide crystal is 5-in. in diameter by 9 in. long

\begin{tabular}{|c|c|c|c|c|c|c|}
\hline$E$ & $\mu$ & $\tau$ & $\pi$ & $\sigma$ & $b$ & $P^{*}$ \\
\hline $\begin{array}{l}\text { Mev } \\
6 \\
10 \\
11 \\
19\end{array}$ & $\begin{array}{c}c m^{2} / g \\
0.0347 \\
.0357 \\
.0371 \\
.0375 \\
.0435\end{array}$ & $\begin{array}{c}c m^{2} / g \\
0.0003 \\
.0002 \\
.0002 \\
.0001 \\
.0001\end{array}$ & $\begin{array}{c}c m^{2} / g \\
0.0156 \\
.0201 \\
.0238 \\
.0255 \\
.0355\end{array}$ & $\begin{array}{c}c m^{2} / g \\
0.0188 \\
.0154 \\
.0131 \\
.0120 \\
.0080\end{array}$ & $\begin{array}{r}0.590 \\
.580 \\
.570 \\
.570 \\
.550\end{array}$ & $\begin{array}{r}0.614 \\
.613 \\
.617 \\
.619 \\
.623\end{array}$ \\
\hline
\end{tabular}

$a$ The values of the absorption coefficients $\mu, \tau, \pi$, and $\sigma$ for sodium iodide were taken from $\mathrm{G}$. White of the Bureau (private communication).

to the extrapolation of $b$ in the evaluation of the 19 -Mev photofraction values should be small.

The extrapolated values of $b$ are given in table 2 together with the modified photofraction, $P^{*}$, from 6 to $19 \mathrm{Mev}$, and the absorption coefficient data corresponding to those in table $1 . P^{*}$ is differentiated from the low energy $p^{*}$ because the latter values for energies above $5 \mathrm{Mev}$ cannot be compared directly with experimental values. $P^{*}$ contains a component $A \pi$, contrasted to $a \pi$ of $p^{*}$, which includes the total area under the pair electron contribution and not just the pair electron area under a Gaussian curve at the photoline position.

In synthesizing the X-ray pulse-height distribution, the Compton scattering events that result in contributions at the photoline energy were included as a Gaussian with an area $b$ and a half-width of $0.09 \sqrt{E}[1,2]$. The area under the Compton electron distribution was, likewise, taken as $(1-b) \sigma$.

\subsection{Contributions Due to the Esc ape of Annihilation Radiation}

The only remaining influence on the broadening of the distribution due to $\mathrm{X}$-rays will result from the escape of one or two annihilation photons from the positron of the electron pair originally produced by the incoming X-ray. Berger and Doggett [2] have calculated the escape of annihilation photons from a 5-in.-diameter by 9-in.-long crystal and found that 63.3 percent of the photons will be completely captured, and 21.5 percent of the events have exactly one annihilation photon escape with no interaction. The remaining 15.2 percent of the events have some interaction of the one or two annihilation photons before some of their energy escapes from the crystal. For the present purposes it is sufficiently accurate to assume that 36.7 percent of the events have exactly one annihilation escape and, therefore, that percentage of the folded electron-pair pulse-height distribution will be displaced downward in energy by 0.51 Mev.

\subsection{Synthesis of the X-Ray Pulse-Height Distributions}

The four contributions discussed above have been used to synthesize the pulse-height distribution for 11- and 19-Mev X-ray photons. The areas of these contributions were all referred to the area under the folded electron-pair distribution. The relative contributions are given in table 3 .

The component distributions are given in figure 9 , a for $11 \mathrm{Mev}$ X-rays and in figure 10 , a for $19 \mathrm{Mev}$ $\mathrm{X}$-rays. The sum of these distributions and the final result of the predictions are given as the solid lines in the $b$ curves of these figures.

TABLE 3. Relative areas of the four contributions to the X-ray pulse-height distribution

\begin{tabular}{|c|c|c|c|}
\hline Contribution & Fraction $(F)$ & $\begin{array}{l}a 11-\mathrm{Mev} \\
\text { value }\left(F_{11}\right)\end{array}$ & $\begin{array}{c}a 19-\mathrm{Mev} \\
\text { value }\left(F_{19}\right)\end{array}$ \\
\hline $\begin{array}{l}\text { Compton events to photoline } \\
\text { Compton distribution } \\
\text { Folded electron pair distri- } \\
\text { bution..... } \\
\text { Secondary annihilation dis- } \\
\text { tribution }\end{array}$ & $\begin{array}{r}b \sigma / a \pi \\
(1-b) \sigma / a \pi \\
a \pi / a \pi \\
(1-a) \pi / a \pi\end{array}$ & $\begin{array}{l}0.423 \\
.319 \\
1 \\
0.580\end{array}$ & $\begin{array}{r}0.195 \\
.160 \\
1 \\
0.580\end{array}$ \\
\hline
\end{tabular}

$a$ Values in table 3 were calculated with data from tables 1 and 2.

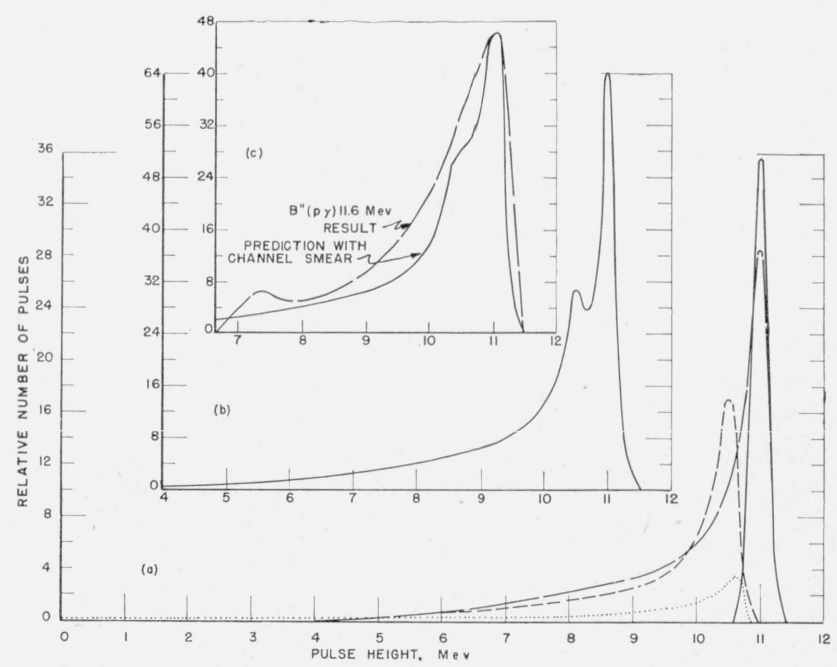

FIGURE 9. Synthesis of the pulse-height distribution produced by 11-Mev X-rays in a 5-in.-diameter by 9-in.-long sodium iodide crystal spectrometer.

(a) Four contributions to the synthesized distribution: (1) Compton electron distribution (dotted eurve); (2) multiple Compton interactions that contribute to the photoline energy (solid curve); (3) folded electron-pair distribution (long dashed curve); (4) secondary distribution due to loss of annihilation radiation (short dashed curve).

(b) Addition of the four curves of (a) to obtain the predicted pulse height distribution.

(c) Comparison of the $11-\mathrm{Mev}$ distribution of (b) with the experimental 11.6 Mev distribution. The distribution of (b) has been smoothed by the channe widths used in obtaining the experimental distribution. The abscissa and ordinate scales of the experimental distribution were normalized to the predicted distribution. 


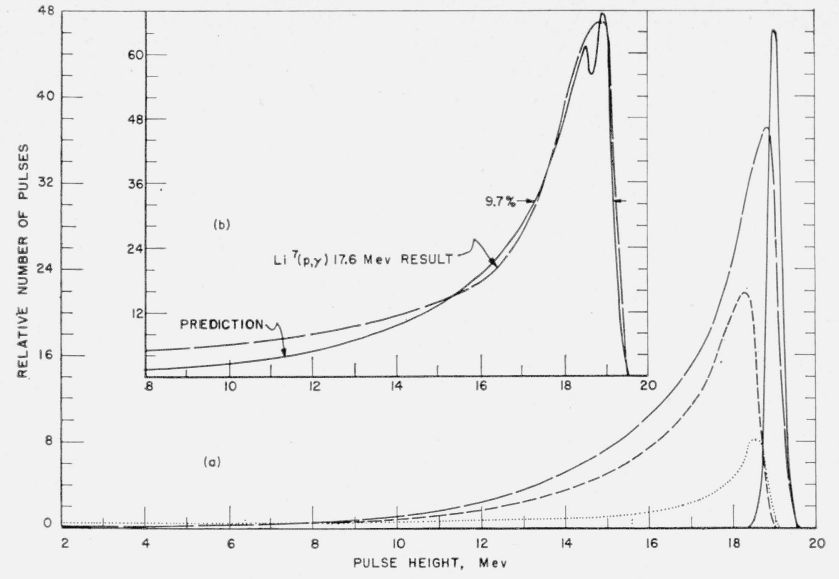

Figure 10. Synthesis of the pulse-height distribution produced by 19-Mev X-rays in a 5-in.-diameter by 9-in.-long sodiumiodide crystal spectrometer.

(a) Four contributions to the synthesized distribution: (1) Compton electron distribution (dotted curve); (2) multiple Compton interactions that contribute to the photoline energy (solid curve); (3) folded electron-pair distribution (long dashed curve); (4) secondary distribution due to loss of annihilation radiation (short dashed curve).

(b) Composite distribution (solid curve) obtained by adding the curves in (a). Comparison is made with the experimental $17.6-\mathrm{Mev}$ distribution. In this case the experimental spectrum was taken with small channel widths so that the case the experinenture the smearing thus introduced was negligible (dashed curve). The abscissa and ordinate scales of the experimental distribution were normalized to the predicted
distribution.

\section{Discussion}

The synthesized pulse-height distributions for 11Mev and 19-Mev X-rays were compared with experimental distributions obtained previously with 11.6- and 17.6-Mev gamma rays [1]

Figure 9,c, shows the experimental distribution for the $\mathrm{B}^{11}(p, \gamma)$ gamma-rays [1]. Because the intensity of this source was small, the channel window size on the differential pulse-height analyzer had to be made large. The curve of figure $9, \mathrm{~b}$, has been smeared with the channel window used in the experiment in order to arrive at the prediction shown as the solid curve of figure 9,c. The experimental and predicted curves have been normalized at the peak. The experimental curve is higher than the predicted one, at pulse heights smaller than the one at which the peak occurs, for practically all pulse heights. However, the differences can be attributed to the Compton scattered gamma rays from the thick copper target assembly, and to the collimator wall effects used in the work described in reference [1]. The wall of the 3/-in.-diameter collimator hole subtended a relatively large solid angle for the gamma rays from the target.
Hence gamma rays could interact and lose energy in the wall and then penetrate into the large-diameter crystal. Both effects of target and collimator would result in degraded-energy gamma rays entering the crystal. Similar comments can be made regarding the comparison in figure $10, \mathrm{~b}$, of the $19-\mathrm{Mev}$ prediction with that obtained with the $17.6-\mathrm{Mev}$ gamma rays from the $\mathrm{Li}^{7}(p, \gamma)$ reaction [1].

No detailed calculation to correct the experimental curves has been made because the experimental data [1], particularly that of the $11.6 \mathrm{-Mev}$ line, were too poor to warrant extensive interpretations. Nevertheless, the comparison between prediction and experiment is considered to be good.

The method of synthesizing pulse-height distributions due to $\mathrm{X}$-rays, which has been outlined, is the best procedure known to the present authors in the X-ray range from 10 to $20 \mathrm{Mev}$. Because of the general success and the expected validity of the predictions, it is planned to apply a similar procedure for energies from 5 to $19 \mathrm{Mev}$ in $2-\mathrm{Mev}$ increments. These results, combined with the work of Berger and Doggett [2], will allow an interpretation of experimental pulse-height distributions in the range from $100 \mathrm{kev}$ to $20 \mathrm{Mev}$, when a total absorption spectrometer with a 5-in. diameter and a 9-in.-long crystal are used.

The work of B. Petree and E. G. Fuller in obtaining a well-controlled electron beam for the present experiment is gratefully acknowledged. Discussions with M. Berger and N. Starfelt were very helpful.

\section{References}

[1] R. S. Foote and H. W. Koch, Rev. Sci. Instr. 25, 746 (1954).

[2] M. J. Berger and J. Doggett, J. Research NBS 56, 355 (1956) RP2686.

[3] H. W. Koch, J. Wyckoff, and B. Petree, Phys. Rev. 99, 663 (1955).

[4] R. S. Foote and Ben Petree, Rev. Sci. Instr. 25, 694 (1954).

[5] E. L. Goldwasser, F. E. Mills, and A. O. Hanson, Phys. Rev. 88, 1137 (1952).

[6] E. Saunders (private communication)

[7] H. W. Koch and R. E. Carter, Phys. Rev. 78, 165 (1950).

[8] W. Heitler, The quantum theory of radiation, $2 \mathrm{~d}$ ed. (Oxford Univ. Press, Oxford, England, 1949).

[9] M. Berger and J. Doggett (private communication).

[10] Ann Nelms, NBS Circular 542 (1953).

Washington, December 5, 1955 . 